NISTIR 7773

\title{
An Application of Combinatorial Methods to Conformance Testing for Document Object Model Events
}

Carmelo Montanez

D. Richard Kuhn

Mary Brady

Richard M. Rivello

Jenise Reyes

Michael K. Powers 
NISTIR 7773

\section{An Application of Combinatorial Methods to Conformance Testing for Document Object Model Events}

Carmelo Montanez

D. Richard Kuhn

Mary Brady

Richard M. Rivello

Jenise Reyes

Michael K. Powers

November, 2010

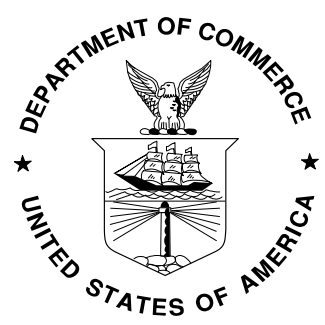

U.S. Department of Commerce

Gary Locke, Secretary 


\section{An Application of Combinatorial Methods \\ to Conformance Testing for Document Object Model Events}

Abstract-This report describes the use of combinatorial test methods to reduce the cost of testing for the Document Object Model Events standard while maintaining an equivalent level of assurance. More than 36,000 tests - all possible combinations of equivalence class values -were reduced by approximately a factor of 20 with no reduction in error detection effectiveness.

\section{Introduction}

The Document Object Model (DOM) [9] is a standardized method for representing and interacting with components of XML, HTML, and XHTML documents. DOM lets programs and scripts access and update the content, structure, and style of documents dynamically, making it easier to produce web applications in which pages are accessed non-sequentially. DOM is standardized by the World Wide Web Consortium (W3C).

Since its origination in 1996 as a convention for accessing and modifying parts of Javascript web pages (known now as DOM Level 0), DOM has evolved as a series of standards offering progressively greater capabilities. Level 1 introduced a model that allowed changing any part of the HTML document, and Level 2 added support for XML namespaces, load and save, cascading style sheets (CSS), traversing the document, and working with ranges of content. Level 3 brings additional features, including keyboard event handling.

DOM Level 3 Events [9] is a W3C Working Draft being written by the Web Applications Working group. Implemented in browsers, it is a generic platform and language neutral event system that allows registration of event handlers, describes event flow through a tree structure, and provides basic contextual information for each event. This work builds on the previous Document Object Model Level 2 events specifications. There are two basic goals in the design of DOM Level 3 Events. The first goal is to design an event system that allows registration of event listeners and describes an event flow through a tree structure. The second goal is to provide a common subset of the current event system used on DOM Level 3 Events browsers.

\section{Constructing Tests for DOM Events}

DOM browser implementations typically contain tens of thousands of source lines of code. To help ensure successful implementations of this complex standard, NIST developed the DOM Conformance Test Suites, which include tests for many DOM components. Early DOM tests were hand-coded in a test language, then processed to produce ECMAScript and Java. In the current version of the test suites, tests are specified in an
XML grammar, allowing easy mapping from specification to a variety of language bindings. Because the grammar is generated automatically [7] from the DOM specs, tests can be constructed quickly and correctly. Output of the test generation process includes the following components, which implementers can use in testing their product for DOM interoperability:

- Tests in the XML representation language,

- $\quad$ XSLT stylesheets necessary to generate the Java and ECMA Script bindings,

- $\quad$ Generated executable code.

Tests for 35 (out of 36) DOM Events were generated. The specification defines each event with an Interface Definition Language (IDL), which in turn defines a number of functions for each event. A typical function can have anywhere from one to fifteen parameters. The API for each function is defined as an XML infoset, which specifies the abstract data model of the XML document using a predefined set of tags. The XML infosets were programmatically generated through a Java application. Since the IDL definition could be accessed directly from the specs web site; the web address was given as input to the Java application. This way the application could read and traverse them extracting just the information of our interest. In this case the function names and their respective parameters, argument names, etc., which became part of the XML file that was used to feed a test generation tool to automatically create the DOM Level 3 tests.

Conventional category partitioning was used to select representative values for non-Boolean parameters. The initial test set was exhaustive across the equivalence classes, producing 36,626 tests that exercised all possible combinations of equivalence class elements. Note that this is not fully exhaustive - all possible value combinations - because such a test suite is generally intractable with continuous range parameters. It is however exhaustive with respect to the equivalence class elements. Thus this test suite will be referred to as the exhaustive test suite in the remainder of this report. Two different implementations were tested. The DOM events and number of tests for each are shown in Table 1. This set of exhaustive tests detected a total of 72 failures. Automated tools made it possible to construct tests programatically, greatly reducing the effort required for testing. However, human intervention is required to run individual tests and evaluate test results, so the conformance testing team sought ways to reduce the number of tests required without sacrificing quality. 


\begin{tabular}{|c|c|c|}
\hline Event Name & Param. & Tests \\
\hline Abort & 3 & 12 \\
\hline Blur & 5 & 24 \\
\hline Click & 15 & 4352 \\
\hline Change & 3 & 12 \\
\hline dblClick & 15 & 4352 \\
\hline DOMActivate & 5 & 24 \\
\hline DOMAttrModified & 8 & 16 \\
\hline DOMCharacterDataModified & 8 & 64 \\
\hline DOMElementNameChanged & 6 & 8 \\
\hline DOMFocusIn & 5 & 24 \\
\hline DOMFocusOut & 5 & 24 \\
\hline DOMNodeInserted & 8 & 128 \\
\hline DOMNodeInsertedIntoDocument & 8 & 128 \\
\hline DOMNodeRemoved & 8 & 128 \\
\hline DOMNodeRemovedFromDocument & 8 & 128 \\
\hline DOMSubTreeModified & 8 & 64 \\
\hline Error & 3 & 12 \\
\hline Focus & 5 & 24 \\
\hline KeyDown & 1 & 17 \\
\hline KeyUp & 1 & 17 \\
\hline Load & 3 & 24 \\
\hline MouseDown & 15 & 4352 \\
\hline MouseMove & 15 & 4352 \\
\hline MouseOut & 15 & 4352 \\
\hline MouseOver & 15 & 4352 \\
\hline MouseUp & 15 & 4352 \\
\hline MouseWheel & 14 & 1024 \\
\hline Reset & 3 & 12 \\
\hline Resize & 5 & 48 \\
\hline Scroll & 5 & 48 \\
\hline Select & 3 & 12 \\
\hline Submit & 3 & 12 \\
\hline TextInput & 5 & 8 \\
\hline Unload & 3 & 24 \\
\hline Wheel & 15 & 4096 \\
\hline Total Tests & & 36626 \\
\hline
\end{tabular}

Table 1. DOM Level 3 Events Tests - Exhaustive

\section{Combinatorial Testing Approach}

Because the DOM test suite had already been applied with exhaustive (across equivalence values) tests against a variety of implementations, it provided a valuable opportunity to evaluate combinatorial testing $[2,3,5,7]$ on real-world software. If results showed that a much smaller test suite could achieve the same level of fault detection, then testing could be done at much less cost in staff time and resources. An obvious critical question in using this approach is - what level of t-way interaction is necessary? Can all faults be detected with 2-way (pairwise) tests, or does the application require 3-way, 4-way or higher strength tests? This work helped to address these questions as well.

To investigate the effectiveness of combinatorial testing, covering arrays of 2-way through 6-way tests were produced, using a tool [7] developed by NIST and the University of Texas Arlington. Using $t$-way combinations can significantly reduce the number of tests as compared with exhaustive. For example, the mousedown event (Figure 1) requires 4,352 tests if all combinations are to be realized. Combinatorial testing reduces the set to 86 tests for 4-way coverage.

Table 2 details the number of parameters and number of tests produced for each of the 35 DOM events, for $t=2$ through 6 . That is, the tests covered all 2-way through 6-way combinations of values. Note that for events with few parameters, the number of tests is the same for the original test suite (Table 1) and combinatorial for various levels of $t$. For example, 12 tests were produced for Abort in the original and also for combinatorial testing at $t=3$ to 6 . This is because producing all $n$-way combinations for $n$ variables is simply all possible combinations of these $n$ variables, and Abort has 3 variables. This situation is not unusual when testing configurations with a limited number of values for each parameter. For nine of the 35 events (two Click events, six Mouse events, and Wheel), all combinations are not covered even with 6-way tests. For these events, combinatorial testing provides a significant gain in efficiency.

\section{Test Results}

Table 2 shows the faults detected for each event. All conditions flagged by the exhaustive test suite were also detected by three of the combinatorial testing scenarios (4, 5 and 6 way testing), which indicates that the implementation faults were triggered by 4-way interactions or less. Pairwise testing would have been inadequate for the DOM implementations, because 2way and 3-way tests detected only $37.5 \%$ of the faults. As can be seen in Table 2, the exhaustive (all possible combinations) tests of equivalence class elements and the 4-way to 6-way combinatorial tests were equally successful in fault detection, indicating that exhaustive testing added no benefit. These findings are consistent with earlier studies $[1,4,8]$ that showed that software faults are triggered by interactions of a small number of variables, for applications in a variety of domains. DOM testing was somewhat unusual in that exhaustive testing of equivalence class elements was possible at all. For most software, too many possible input combinations exist to cover even a tiny fraction of the exhaustive set, so combinatorial methods may be of greater benefit for these. 


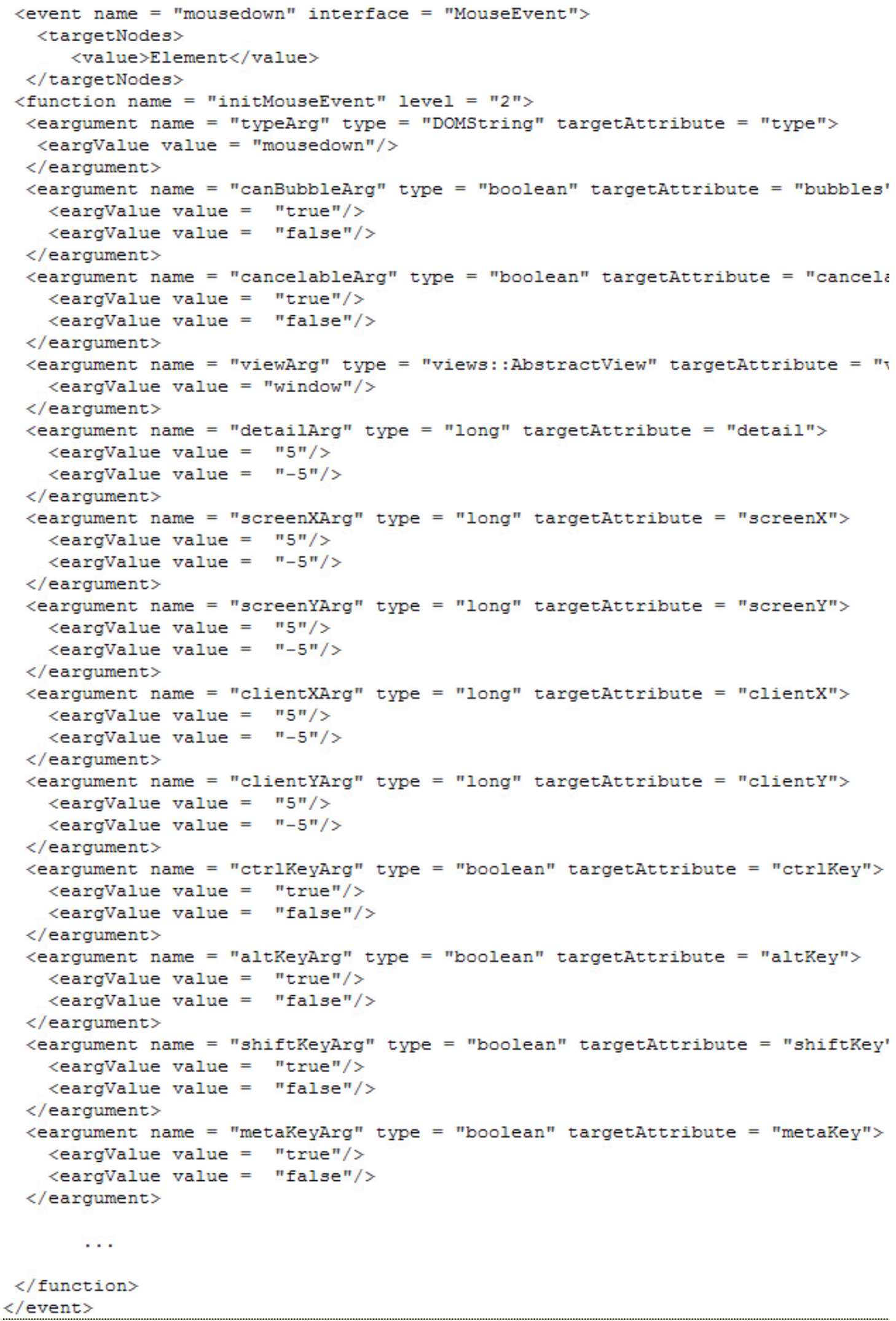

Figure 1. XML infosets generated from IDLs used as input to Test Accelerator. 


\begin{tabular}{|c|c|c|c|c|c|c|}
\hline Event Name & $\begin{array}{l}\text { Num } \\
\text { param }\end{array}$ & $\begin{array}{l}\text { 2-way } \\
\text { Tests }\end{array}$ & $\begin{array}{l}\text { 3-way } \\
\text { Tests }\end{array}$ & $\begin{array}{l}\text { 4-way } \\
\text { Tests }\end{array}$ & $\begin{array}{l}5 \text {-way } \\
\text { Tests }\end{array}$ & $\begin{array}{l}\text { 6-way } \\
\text { Tests }\end{array}$ \\
\hline Abort & 3 & 8 & 12 & 12 & 12 & 12 \\
\hline Blur & 5 & 10 & 16 & 24 & 24 & 24 \\
\hline Click & 15 & 18 & 40 & 86 & 188 & 353 \\
\hline Change & 3 & 8 & 12 & 12 & 12 & 12 \\
\hline dblClick & 15 & 18 & 40 & 86 & 188 & 353 \\
\hline DOMActivate & 5 & 10 & 16 & 24 & 24 & 24 \\
\hline DOMAttrModified & 8 & 8 & 16 & 16 & 16 & 16 \\
\hline DOMCharacterDataModified & 8 & 32 & 62 & 64 & 64 & 64 \\
\hline DOMElementNameChanged & 6 & 8 & 8 & 8 & 8 & 8 \\
\hline DOMFocusIn & 5 & 10 & 16 & 24 & 24 & 24 \\
\hline DOMFocusOut & 5 & 10 & 16 & 24 & 24 & 24 \\
\hline DOMNodeInserted & 8 & 64 & 128 & 128 & 128 & 128 \\
\hline DOMNodeInsertedIntoDocument & 8 & 64 & 128 & 128 & 128 & 128 \\
\hline DOMNodeRemoved & 8 & 64 & 128 & 128 & 128 & 128 \\
\hline DOMNodeRemovedFromDocument & 8 & 64 & 128 & 128 & 128 & 128 \\
\hline DOMSubTreeModified & 8 & 32 & 64 & 64 & 64 & 64 \\
\hline Error & 3 & 8 & 12 & 12 & 12 & 12 \\
\hline Focus & 5 & 10 & 16 & 24 & 24 & 24 \\
\hline KeyDown & 1 & 9 & 17 & 17 & 17 & 17 \\
\hline KeyUp & 1 & 9 & 17 & 17 & 17 & 17 \\
\hline Load & 3 & 16 & 24 & 24 & 24 & 24 \\
\hline MouseDown & 15 & 18 & 40 & 86 & 188 & 353 \\
\hline MouseMove & 15 & 18 & 40 & 86 & 188 & 353 \\
\hline MouseOut & 15 & 18 & 40 & 86 & 188 & 353 \\
\hline MouseOver & 15 & 18 & 40 & 86 & 188 & 353 \\
\hline MouseUp & 15 & 18 & 40 & 86 & 188 & 353 \\
\hline MouseWheel & 14 & 16 & 40 & 82 & 170 & 308 \\
\hline Reset & 3 & 8 & 12 & 12 & 12 & 12 \\
\hline Resize & 5 & 20 & 32 & 48 & 48 & 48 \\
\hline Scroll & 5 & 20 & 32 & 48 & 48 & 48 \\
\hline Select & 3 & 8 & 12 & 12 & 12 & 12 \\
\hline Submit & 3 & 8 & 12 & 12 & 12 & 12 \\
\hline TextInput & 5 & 8 & 8 & 8 & 8 & 8 \\
\hline Unload & 3 & 16 & 12 & 24 & 24 & 24 \\
\hline Wheel & 15 & 20 & 44 & 92 & 214 & 406 \\
\hline Total Tests & & 702 & 1342 & 1818 & 2742 & 4227 \\
\hline
\end{tabular}

Table 2. DOM 3 Level Tests - Combinatorial 


\begin{tabular}{|l|l|l|l|l|l|}
\hline \multirow{2}{*}{$\boldsymbol{t}$} & \multirow{2}{*}{ Tests } & \multirow{2}{*}{$\begin{array}{l}\text { \% of } \\
\text { Original }\end{array}$} & \multicolumn{3}{|l|}{ Test Results } \\
\cline { 4 - 6 } & & & Pass & Fail & Not Run \\
\hline 2 & 702 & $1.92 \%$ & 202 & 27 & 473 \\
\hline 3 & 1342 & $3.67 \%$ & 786 & 27 & 529 \\
\hline 4 & 1818 & $4.96 \%$ & 437 & 72 & 1309 \\
\hline 5 & 2742 & $7.49 \%$ & 908 & 72 & 1762 \\
\hline 6 & 4227 & $11.54 \%$ & 1803 & 72 & 2352 \\
\hline
\end{tabular}

Table 3. Comparison of t-way with exhaustive test set size.

The original test suite contained a total of 36,626 tests (Table 1) for all combinations of events, but after applying combinatorial testing, the set of tests is dramatically reduced depending on the $t$-way interactions algorithm, as shown in Table 3. It is important to note that combinatorial testing is constrained by some of the same limitations as other test methodologies. In particular, for most testing some method of abstracting parameter values, such as equivalence classes, must be used. The number of tests generated in combinatorial covering arrays is proportional to $v^{t} \log n$, for $t$-way interactions where each of $n$ parameters has $v$ values. In cases where most parameters have a small number of discrete values, this is less of a limitation, but it was required for parameters such as screen $\mathrm{X}$ and $\mathrm{Y}$ values, and must be considered for most software testing.

Table 2 also shows results for 2-way through 6-way testing. Notice that although the number of tests that successfully execute varies from $t$-way combination to $t$-way combination, the number of failures remains a constant at $t=2$ and 3 , and at $t=4$ to 6 . The last column shows the tests that did not execute to completion, in almost all cases due to non-support of the feature under test.

DOM results were consistent with previous findings that testing a small number of interactions (in this case 4-way) was sufficient to detect all errors. Comparing results of the DOM testing with previous data $[1,4]$ on $t$-way interaction failures (Figure 2), we can see that some DOM failures were more difficult to detect, in the sense that a smaller percentage of the total were found by 2-way and 3-way tests than for the other application domains. The unusual shape of the curve for DOM tests may result from the large number of parameters for which exhaustive coverage was reached (so that the number of tests remained constant after a certain point). There are thus two sets of events: a large set with few possible values which could be covered exhaustively with 2-way or 3-way tests, and a smaller set with a larger input space (from 1024 to 4352). In particular, nine events (click, dblClick, mouse events, and wheel) all have the same input space size, with number of tests increasing at the same rate for each, while for the rest, exhaustive coverage is reached at either $t=2$ or $t=3$. The ability to compare results of previously-conducted exhaustive testing with combinatorial testing provides an added measure of confidence in the applicability of these methods to this type of interoperability testing.

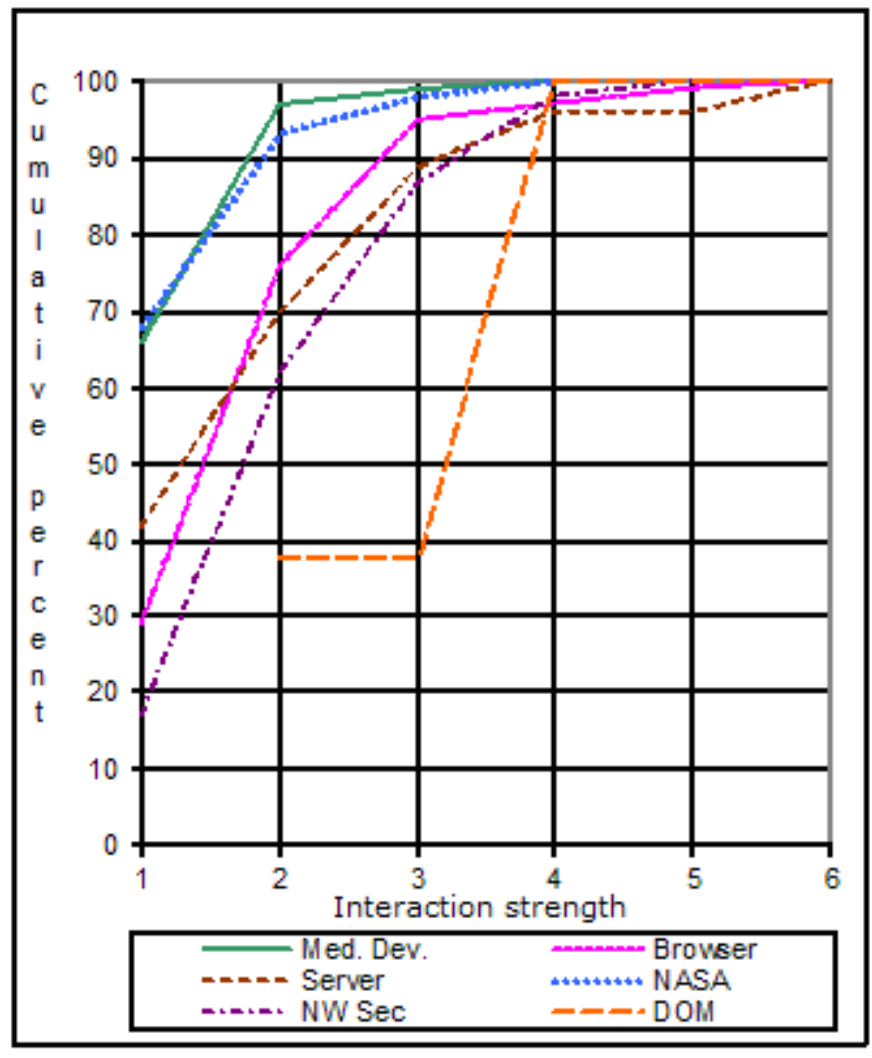

FIGURE 2. DOM COMPARED WITH OTHER APPLICATIONS

\section{Conclusions}

The DOM Events testing suggests that combinatorial testing can significantly reduce the cost and time required for conformance testing for standards with characteristics similar to DOM. What is the appropriate interaction strength to use in this type of testing? Intuitively, it seems that if no additional faults are detected by $t$-way tests, then it may be reasonable to conduct additional testing only for $t+1$ interactions, but no greater if no additional faults are found at $t+1$. In empirical studies of software failures, the number of faults detected at $t>$ 2 decreased monotonically with $t$, and the DOM testing results are consistent with this earlier finding. Following this strategy for the DOM testing would result in running 2-way tests through 5-way, then stopping because no additional faults were detected beyond the 4-way testing. Alternatively, given the apparent insufficient fault detection of pairwise testing (see Figure 2), testers may prefer to standardize on a 4-way or higher level of interaction coverage. This option may be particularly attractive for an organization that produces a series of similar products and has enough experience to identify the most cost-effective level of testing. Even the relatively strong 4 -way testing in this example was only $5 \%$ of the original test set size. Results in this study have been sufficiently promising for combinatorial methods to be applied in testing other interoperability standards. 


\section{References}

1. K.Z. Bell, Optimizing Effectiveness and Efficiency of Software Testing: a Hybrid Approach, PhD Dissertation, North Carolina State University, 2006.

2. Bryce, R. C.J. Colbourn, M.B. Cohen. A Framework of Greedy Methods for Constructing Interaction Tests. The $27^{\text {th }}$ International Conference on Software Engineering (ICSE), St. Louis, Missouri, pages 146-155. (May 2005).

3. Grindal, Mats, Offutt, Jeff, and Andler, Sten F. "Combination Testing Strategies: A Survey," Journal of Software Testing, Verification and Reliability vol. 15, no. 3, pp. 167-199, 2005.

4. Kuhn, D. R., D. Wallace, and A. Gallo, "Software Fault Interactions and Implications for Software Testing,"

IEEE Transactions on Software Engineering, 30(6):418-421, 2004.

5. Lei, Y. K.C. Tai. In-parameter order: a Test Generation Strategy for Pairwise Testing. Proceedings of the Third IEEE High Assurance Systems Engineering Symposium, pp. 254-261, IEEE, Nov. 1998.

6. National Institute of Standards and Technology. Test Accelerator. http://www.itl.nist.gov/div897/docs/testacc.html

7. National Institute of Standards and Technology. Automated Combinatorial Testing for Software. http://csrc.nist.gov/acts

8. Wallace, D.R. D.R. Kuhn. "Failure Modes in Medical Device Software: an Analysis of 15 Years of Recall Data”, International Journal of Reliability, Quality, and Safety Engineering, Vol. 8, No. 4, 2001.

9. World Wide Web Consoritum, DOM Level 3 Events Specification, 8 Sept 2009. http://www.w3.org/TR/DOMLevel-3-Events/

Acknowledgments: We are very grateful to Vadim Okun for a careful review and for suggesting some possible explanations for the fault detection rate at various $t$ levels in the DOM testing.

Disclaimer: We identify certain software products in this document, but such identification does not imply recommendation by the US National Institute for Standards and Technology, nor does it imply that the products identified are necessarily the best available for the purpose. 
This page was intentionally left blank. 